\title{
Quantification and mitigation of the impact of scene inhomogeneity on Sentinel-4 UVN UV-VIS retrievals
}

\author{
S. Noël ${ }^{1}$, K. Bramstedt ${ }^{1}$, H. Bovensmann ${ }^{1}$, K. Gerilowski ${ }^{1}$, J. P. Burrows ${ }^{1}$, C. Standfuss ${ }^{2}$, E. Dufour ${ }^{2}$, and \\ B. Veihelmann ${ }^{3}$ \\ ${ }^{1}$ Institute of Environmental Physics, University of Bremen, FB 1, P.O. Box 330440, 28334 Bremen, Germany \\ ${ }^{2}$ Noveltis, Parc Technologique du Canal, 2, avenue de l'Europe, 31520 Ramonville-St Agne, France \\ ${ }^{3}$ ESA/ESTEC, Keplerlaan 1, 2201 AZ Noordwijk, The Netherlands
}

Correspondence to: S. Noël (stefan.noel@iup.physik.uni-bremen.de)

Received: 10 February 2012 - Published in Atmos. Meas. Tech. Discuss.: 1 March 2012

Revised: 7 May 2012 - Accepted: 17 May 2012 - Published: 11 June 2012

\begin{abstract}
The quality of trace gas products derived from measurements of a space-borne imaging spectrometer is affected by the inhomogeneity of the illumination of the instrument slit and thus by the heterogeneity of the observed scene. This paper aims to quantify this effect and summarise findings on how to mitigate the impact of inhomogeneous slit illumination on tropospheric $\mathrm{O}_{3}, \mathrm{NO}_{2}, \mathrm{SO}_{2}$ and $\mathrm{HCHO}$ columns derived from measurements of the Sentinel-4 UVN imaging spectrometer. For this purpose, spectra for inhomogeneous ground scenes have been simulated based on a combination of a radiative transfer model and spatially high resolved MODIS (Moderate Resolution Imaging Spectroradiometer) data. The resulting errors on tropospheric $\mathrm{O}_{3}$, $\mathrm{NO}_{2}, \mathrm{SO}_{2}$ and $\mathrm{HCHO}$ columns derived from these spectra have been determined via an optimal estimation approach. We conclude that inhomogeneous illumination results in significant errors in the data products if the natural inhomogeneity of the observed scenes are not accounted for. $\mathrm{O}_{3}$ columns are less affected than the other data products; largest errors occur for $\mathrm{NO}_{2}$ (mean absolute errors about $5 \%$, maximum error exceeding $50 \%$, standard deviation of the errors about $8 \%$ ). These errors may be significantly reduced (by factors up to about 10) by an appropriate wavelength calibration applied individually to each Earthshine radiance spectrum. With wavelength calibration the estimated mean absolute errors due to inhomogeneity are for all gases well below $1 \%$; standard deviations of the errors are $1.5 \%$ or lower; maximum errors are about $10 \%$ for $\mathrm{NO}_{2}$ and around $5 \%$ for the other gases.
\end{abstract}

\section{Introduction}

Light entering an imaging spectrometer is spectrally dispersed along one of the spatial dimensions of the scene that is seen through the telescope. Depending on the heterogeneity of the observed scene, the entrance slit of the spectrometer will be inhomogeneously illuminated in both the spectral and spatial direction which results in a scene dependent slit function. With "slit function" we denote here the spectral response function, i.e. the instrument response in the spectral domain. This variable slit function, if not taken properly into account, will affect the spectral calibration of the sensor (Voors et al., 2006) and will introduce a pseudo noise component into the measured top-of-atmosphere reflectance (Earth radiance over solar irradiance). This pseudo-noise will then affect the quality of trace gas products derived from the reflectance spectra using absorption spectroscopic techniques like for example the Differential Optical Absorption Spectroscopy (DOAS, see, e.g. Perner and Platt, 1979; Burrows et al., 1999), as these techniques ask for reflectance spectra with high signal-to-noise ratios.

Voors et al. (2006) investigated the impact of scene inhomogeneity, mainly due to clouds, on the spectral calibration of the Ozone Monitoring Instrument (OMI) on Aura (Levelt et al., 2006), which has a spatial resolution of up to $13 \times 24 \mathrm{~km}^{2}$ (for the nadir pixels). They showed that inhomogeneous slit filling due to inhomogeneous scenes (clouds, etc.) results in wavelength shifts of up to a half spectral pixel, corresponding to $0.07 \mathrm{~nm}$ in the UV2 and $0.10 \mathrm{~nm}$ in the VIS band. They concluded that with an adequate spectral

Published by Copernicus Publications on behalf of the European Geosciences Union. 
calibration approach, the impact of the inhomogeneous slit illumination on spectral calibration can be minimised. Gerilowski et al. (2011) demonstrated with an airborne spectrometer that scene inhomogeneities on scales of about $50 \mathrm{~m}$ result in enhanced noise contributions even under cloud free conditions. Nevertheless, there is to the authors' knowledge no analysis of impact of scene inhomogeneity on the trace gas concentrations errors published so far. Therefore, this paper aims to quantify the impact and summarise findings on how to mitigate the impact of inhomogeneous slit illumination on trace gas concentrations. Specifically, the present study concentrates on measurements of weak absorbers in the UV-VIS spectral region performed by the Sentinel-4 UVN instrument.

The Sentinel-4 UVN instrument (Bazalgette CourrègesLacoste et al., 2011; Ahlers et al., 2011) is an imaging spectrometer designed to monitor air quality over Europe from geostationary orbit. It has been developed for the European Union programme Global Monitoring of Environment and Security (GMES). Sentinel-4 UVN is being constructed by ESA as part of the core payload of the Meteosat Third Generation, MTG, which is an operational meteorological satellite system managed by EUMETSAT. The Sentinel-4 UVN instrument builds on the heritage of the Scanning Imaging Absorption Spectrometer for Atmospheric Chartography (SCIAMACHY, see, e.g. Bovensmann et al., 1999) and the GeoSCIA concepts (see, e.g. Bovensmann et al., 2002, 2004). The main purpose of the Sentinel-4 mission is to monitor the air quality by measurements of tropospheric $\mathrm{O}_{3}$, $\mathrm{NO}_{2}, \mathrm{SO}_{2}, \mathrm{HCHO}$ and aerosol quantities. Sentinel-4 UVN is currently under development. The Sentinel-4 mission will consist of two instruments, the first one to be launched in 2018 on board the Meteosat Third Generation satellite MTGS1. Similar to OMI, UVN will use 2-dimensional CCD detectors. It measures direct as well as backscattered solar irradiance in two spectral bands, the UV-VIS $(305-500 \mathrm{~nm}$, spectral resolution $0.5 \mathrm{~nm})$ and the NIR $(750-775 \mathrm{~nm}$, spectral resolution $0.12 \mathrm{~nm}$ ). The long side of the instrument slit is oriented in $\mathrm{N}-\mathrm{S}$ direction. Continuous scans in $\mathrm{E}-\mathrm{W}$ direction are performed to obtain a spectrally resolved image of Europe on an hourly scale.

The typical UVN ground pixel size over Europe is $8 \mathrm{~km} \times 8 \mathrm{~km}$, which is considerably smaller than currently operational similar Earth atmosphere observation instrumentation in low-earth orbits. The spatial response function of the UVN instrument in east-west direction is shown in Fig. 1, giving the sensitivity of the instrument to light coming from different directions/spatial distances relative to the centre of the observed scene. As can be seen from this figure, the spatial response function is in the UV-VIS of trapezoidal (almost rectangular) shape with a full width at half maximum (FWHM) of about $8 \mathrm{~km}$. During one integration time (about $6 \mathrm{~s}$ ), the instantaneous field of view (IFOV) is moved about $8 \mathrm{~km}$ from the east to the west by the scan. By this the spatial

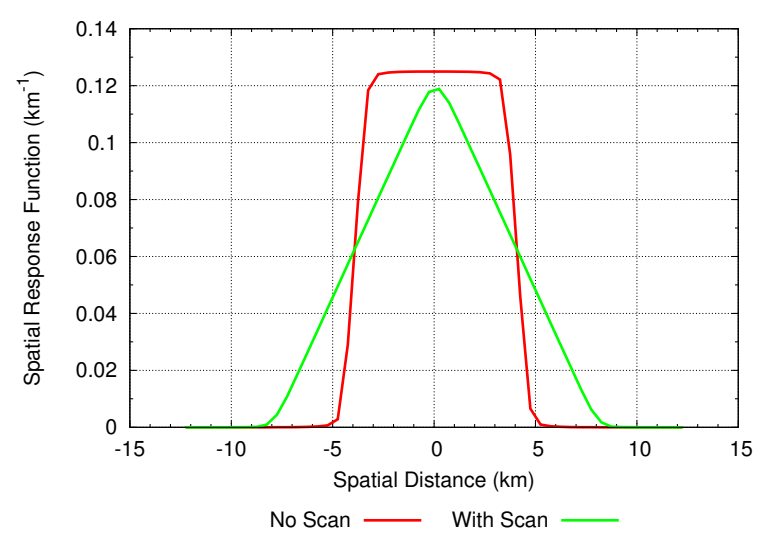

Fig. 1. Normalised spatial response function for the UV-VIS in east-west direction in ground coordinates. Red: without scan. Green: with scan.

response function is smeared, resulting in an almost triangular shape (but with similar FWHM).

The natural heterogeneity of such a ground scene, arising from different surface albedo or cloudiness, results in an inhomogeneous illumination of the instrument slit, which in turn alters the instrument spectral response function (ISRF). Since this ISRF depends on the actual scene, it is highly variable and usually not known, but simultaneous measurements of higher spatial resolution may enable this ISRF to be inferred. Effectively, the inhomogeneous illumination generates an additional error if it is not accounted for in the retrieval, i.e. if in the retrieval a homogeneous illumination is assumed.

This manuscript describes investigations performed to assess and mitigate the impact of inhomogeneous illumination of the instrument slit on the Sentinel-4 UVN UV-VIS data products $\mathrm{O}_{3}, \mathrm{NO}_{2}, \mathrm{SO}_{2}$ and $\mathrm{HCHO}$. Although the simulations shown in this manuscript have been specifically performed for the UVN UV-VIS instrumental configuration, the problem of inhomogeneous illumination and thus also possible mitigation strategies are also relevant for other missions using similar instrumentation, like the forthcoming Sentinel5 and its precursor (with the TROPOMI instrument). However, the UVN UV-VIS band has the advantage that it has been designed such that the spectrometer's optical bench has almost no smile, i.e. the spectral calibration does not vary in spatial direction. In this case the impact of scene inhomogeneities in the spatial direction of the slit may be neglected and it is sufficient to consider only the inhomogeneous illumination along the spectral direction of the slit, as we do in this study. In this sense the results presented here are specific to the UVN UV-VIS design. This must be taken into account when transferring them to other instruments or spectral bands. 
Table 1. Aerosol settings, based on Lowtran (moderate aerosol).

\begin{tabular}{ll}
\hline Season & Fall/winter \\
Boundary layer aerosol type & Rural \\
Boundary layer visibility & $23 \mathrm{~km}$ \\
Boundary layer humidity & $80 \%$ \\
Tropospheric visibility & $23 \mathrm{~km}$ \\
Tropospheric humidity & $80 \%$ \\
Stratospheric aerosol loading & Background \\
Stratospheric aerosol type & Background \\
Mesospheric aerosol loading & Normal mesosphere \\
\hline
\end{tabular}

\section{Approach}

The overall approach to determine the errors of the tropospheric columns is as follows:

1. Compute a spectrally high resolved reference radiance using a radiative transfer model;

2. Simulate measured radiances for inhomogeneous scenes by convolution of the reference radiance with simulated inhomogeneous ISRFs, i.e. ISRFs derived for a heterogeneous scene as described below;

3. Simulate a measured irradiance by convolution of a reference irradiance spectrum with the corresponding homogeneous ISRF, i.e. an ISRF for homogeneous illumination;

4. (Optionally) apply a wavelength calibration;

5. Calculate the reflectance; and

6. Estimate systematic errors of data products assuming a retrieval with homogeneous ISRF.

Steps 2 to 6 are performed for a set of 400 UVN ground pixels covering an area of $160 \mathrm{~km} \times 160 \mathrm{~km}$.

The following subsections summarise the input quantities and specific algorithms used in this study. More detailed information is given in the Appendix.

\subsection{Radiance and irradiance spectra}

The spectrum of Dobber et al. (2008) is used as irradiance reference spectrum. The radiance reference spectrum is derived by radiative transfer calculations using SCIATRAN 2.2 (Rozanov et al., 2005).

The following geophysical scenario has been assumed: Satellite position $0^{\circ} \mathrm{N}, 0^{\circ} \mathrm{E}, 35786 \mathrm{~km}$ height, 23 September, 15:00 LT, latitude $50^{\circ} \mathrm{N}$, surface albedo 0.05 (spectrally constant). The assumed aerosol settings and columns of trace gases are given in Tables 1 and 2. The tropospheric ozone column corresponds to typical background conditions; for the minor trace gases polluted conditions are assumed. This scenario is considered to be typical for the most interesting UVN measurement conditions. In fact, it is in line with
Table 2. Trace gases columns.

\begin{tabular}{lcc}
\hline $\begin{array}{l}\text { Trace } \\
\text { gas }\end{array}$ & $\begin{array}{c}\text { Tropospheric column } \\
\left(\mathrm{mol} \mathrm{cm}^{-2}\right)\end{array}$ & $\begin{array}{c}\text { Total column } \\
\left(\mathrm{mol} \mathrm{cm}^{-2}\right)\end{array}$ \\
\hline $\mathrm{O}_{3}$ & $6.4 \mathrm{e}+17$ & $9.19 \mathrm{e}+18$ \\
$\mathrm{NO}_{2}$ & $1.0 \mathrm{e}+16$ & $1.58 \mathrm{e}+16$ \\
$\mathrm{SO}_{2}$ & $9.1 \mathrm{e}+16$ & $9.18 \mathrm{e}+16$ \\
$\mathrm{HCHO}$ & $3.0 \mathrm{e}+16$ & $3.10 \mathrm{e}+16$ \\
\hline
\end{tabular}

the scenario used in the UVN signal-to-noise specifications. Note that the results presented in this study do not depend much on the chosen scenario as long as this scenario is consistently used in both forward model and retrieval.

\subsection{Calculation of ISRFs}

Simulated ISRFs for homogeneous and inhomogeneous scenes have been derived based on MODIS (Moderate Resolution Imaging Spectroradiometer, see http://modis.gsfc. nasa.gov) data. For this study, MODIS/Terra data from spectral band 3 (459-479 nm) have been used. In this band, MODIS data have a spatial sampling of $0.5 \mathrm{~km}$ which is considerably higher than the UVN spatial sampling of $8 \mathrm{~km}$ and thus allows the estimation of intensity variations over the UVN slit.

The method to derive these ISRFs is explained in Appendix A.

For the results presented in this manuscript, a typical MODIS scene over land (relatively clear region over Spain, 17 June 2009) has been selected. This scene has been chosen because it contains many cloud-free ground pixels (providing the best situation to derive useful tropospheric information), but also pixels with higher cloud fraction, adding some more variability in the signal (see Fig. 2). The scene covers an area of $160 \mathrm{~km} \times 160 \mathrm{~km}$, which corresponds to 400 $(20 \times 20)$ UVN ground pixels. ${ }^{1}$ This number is considered to be sufficient to derive statistically meaningful results.

In the context of this study, the so-called "reflectance ratio" (RR) is used to characterise the inhomogeneity of a ground pixel. The reflectance ratio is defined as:

$\mathrm{RR}=\frac{L_{\text {left }}}{L_{\text {right }}}$,

where $L_{\text {left }}$ denotes the sum of all sub-pixel reflectances left of the centre of the field of view/slit and $L_{\text {right }}$ is the sum of all sub-pixel reflectances right of the centre. The reflectance ratio is determined from sub-pixel reflectance data also derived from MODIS.

\footnotetext{
${ }^{1}$ The Sentinel-4 spatial sampling distance (SSD) has been assumed constant $(8 \mathrm{~km})$ for simplicity. Variations of the SSD, e.g. due to the projection on the Earth's surface, have been ignored. The assumed SSD of $8 \mathrm{~km}$ is in line with the requirement at a reference location at $45^{\circ} \mathrm{N}$.
} 


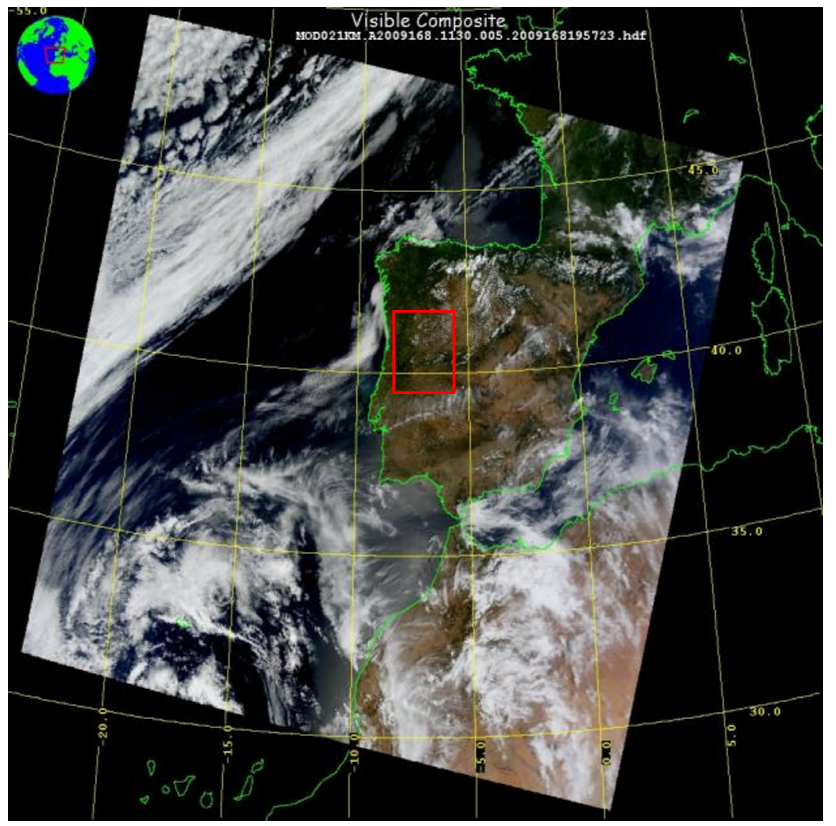

Fig. 2. Visible composite of MODIS/Terra data for 17 June 2009, 11:30-11:35 UT. Source: LAADS. The red square indicates the approximate position of the chosen region.

Figure 3 shows the spatial distribution of the cloud fraction for the selected scene as well as the mean and standard deviation of the sub-pixel reflectances and the derived reflectance ratios. The cloud fraction is approximated by the reflectance variation between clear-sky and overcast threshold values derived empirically such that the average cloud fraction is consistent with the MODIS cloud cover product (at $5 \mathrm{~km}$ sampling) over the entire scene. Therefore, the spatial distribution of cloud fraction and reflectance is fully correlated. As expected, mean and standard deviation of the radiances typically correlate well with the cloud fraction; the reflectance ratio deviation from one is usually high at large cloud or reflectance gradients.

The determination of cloud fractions based on a combination of (visible) reflectances and cloud fractions from thermal infrared data may result in too high values as a result of the larger influence of cirrus clouds in the thermal infrared. However, as explained below, the derived cloud fractions are not used in the error mapping procedure; they are only used to define a sub-group of results (in this case those for cloud fractions $<20 \%$ ), which is considered to be most appropriate to derive tropospheric information. This threshold of $20 \%$ is based on experience from other instruments/retrievals and has to be verified as soon as real UVN data are available. Therefore, the absolute accuracy of the derived cloud fractions is considered to be not critical for the present study.

The reflectance ratio is also useful to classify the derived inhomogeneous ISRFs, as can be seen from Fig. 4. ISRFs with a reflectance ratio close to 1 are symmetric and very similar to the homogeneous ISRF. The ISRFs become more asymmetric when the reflectance ratio deviates from 1 .

\subsection{Spectral calibration algorithm}

The spectral calibration has been performed using a newly developed algorithm, which is described in detail in Appendix B. The reference spectra used in the spectral calibration have been convoluted with the homogeneous ISRF. The spectral calibration algorithm uses as weights errors which have been derived from expected UVN signal-to-noise ratios (SNRs) shown in Fig. 5.

The spectral calibration algorithm used here differs in some respect from the one used in OMI operational processing (Voors et al., 2006). For OMI, there are two independent algorithms: one for spectral assignment and one for spectral calibration. The spectral calibration is determined for a number of irradiance spectra obtained at a reference temperature of the optical bench. This is done by fitting a reference solar spectrum to the measured irradiances. The spectral assignment is based on a fixed set of polynomials per ground pixel (providing a wavelength per pixel) and correction parameters to these polynomial parameters for optical bench temperature dependence and non-homogeneous illumination of the instrument entrance slit. The scene inhomogeneity is derived from measurements with higher spatial (temporal) sampling at specific wavelengths. The polynomial correction parameters are derived from comparing the in-flight spectral assignment and spectral calibration data.

The wavelength calibration algorithm used in the present study is in fact very similar to the OMI spectral calibration in the sense that in both cases absorption features are fitted to the measured spectra. However, in the present case the spectral calibration fit is applied to each individual radiance and irradiance spectrum without sub-pixel knowledge instead of using a-posteriori corrections.

\subsection{Reflectance calculation algorithm}

The UVN instrument will measure the spectral radiance $R$ and the spectral irradiance $I$ as functions of wavelength $\lambda$. The sun normalised radiance $L$ is defined as the ratio of radiance to irradiance:

$L:=\frac{R}{I}$.

In the context of this study, $L$ is equivalent to a reflectance that is usually defined in a similar way but includes additional geometrical factors, like the cosine of the solar zenith angle. This difference is however not relevant here because these factors do not have a spectral dependence.

The problem is that radiance and irradiance are usually measured on slightly different wavelength grids. Reasons for these different wavelength grids are different Doppler shifts resulting from different viewing directions, small thermal changes of the instrument between the measurements or 

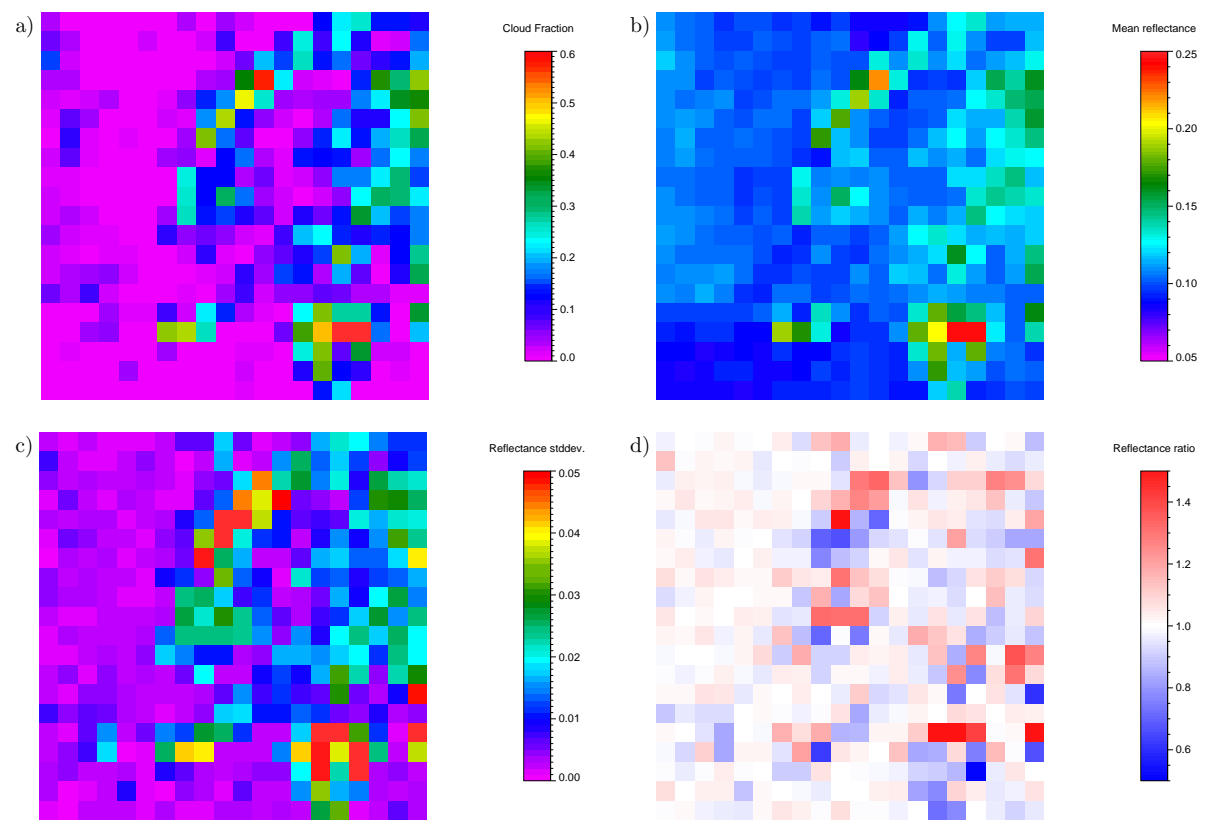

Fig. 3. Spatial distribution of reflectance quantities for the selected scene. Each square in the sub-figures correspond to one UVN ground pixel. (a) Cloud fraction (calculated as described in Sect. 2.2), (b) mean sub-pixel reflectances, (c) standard deviation of sub-pixel reflectances, (d) reflectance ratios (as defined in Eq. 1).
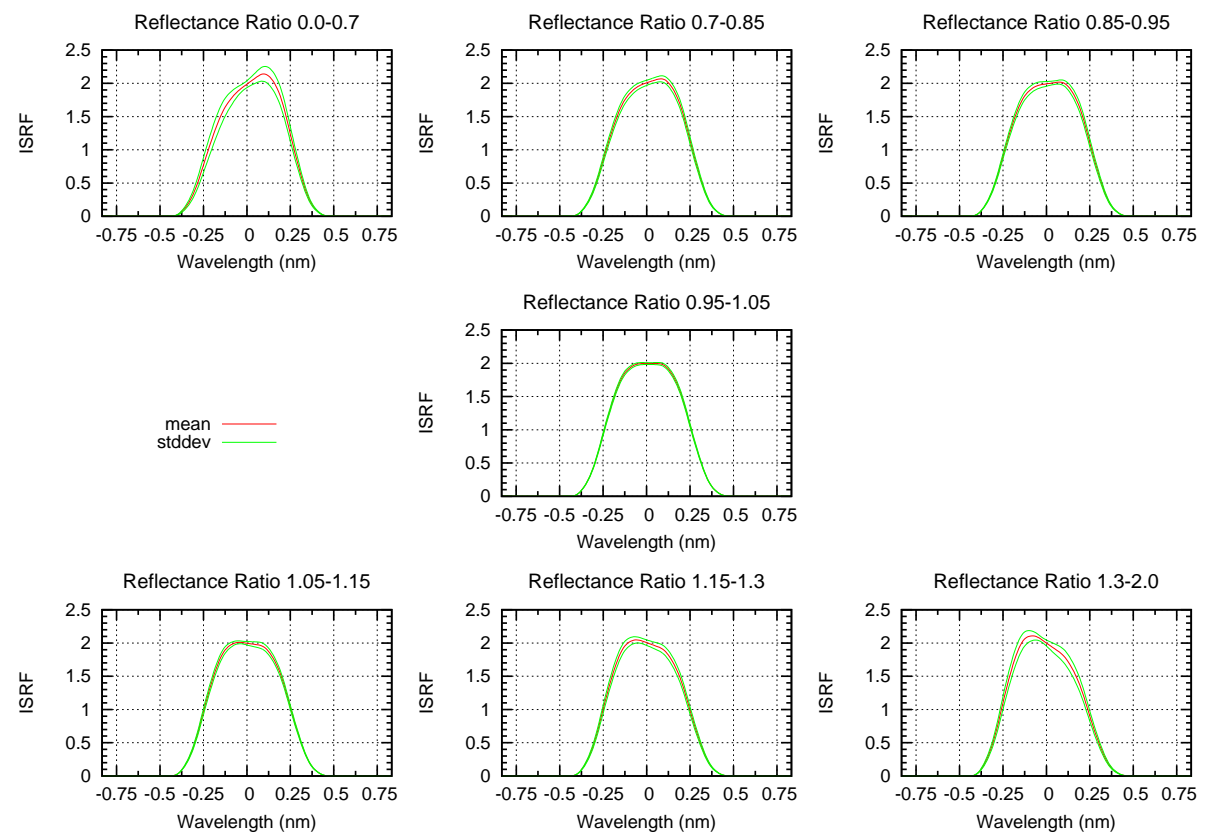

Fig. 4. Classification of inhomogeneous ISRFs. Red: mean ISRFs for the specified interval of reflectance ratios. Green: plus/minus corresponding standard deviation.

potentially slightly different illumination conditions (as addressed in the present paper). Therefore, an interpolation is required. As the variability of irradiances is smaller than the variability of radiances, the preferred way is to interpolate the irradiance to the radiance spectral grid.
Different methods can be used for the interpolation. The simplest way is to perform a linear interpolation, but this may cause errors if the spectra are not strongly oversampled (which is not the case for UVN spectra, where the sampling ratio is typically 3 ). A better method, which is quite 


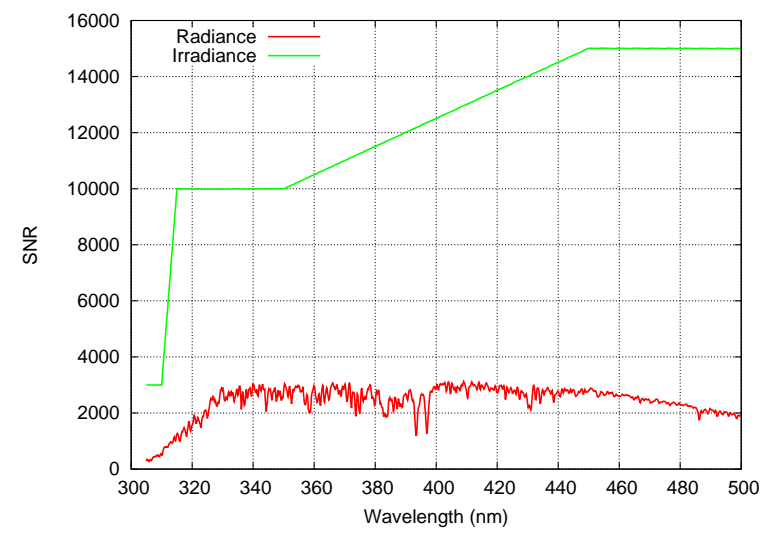

Fig. 5. Expected signal-to-noise ratios of the UVN instrument in the UV-VIS band. The irradiance SNR shown is the requirement.

commonly used also for spectral data from other instruments, is a spline interpolation.

In the present case we use the so-called "high sampling interpolation method" (further on abbreviated with HSM), which has been developed by Pepijn Veefkind from KNMI (personal communication, 2010) to determine reflectances out of radiances and irradiances. These are then later used in the error mapping.

The HSM uses additional information from a solar reference spectrum with high sampling $\left(I_{\text {ref }}\right)$. For the HSM, the irradiance at a spectral position $\lambda_{j}$ is given by:

$I\left(\lambda_{j}\right)=I\left(\lambda_{k}\right) \frac{I_{\mathrm{ref}}\left(\lambda_{j}\right)}{I_{\mathrm{ref}}\left(\lambda_{k}\right)}$,

where $\lambda_{k}$ is the nearest neighbour spectral pixel of $\lambda_{j}$ and $I_{\text {ref }}$ is linearly interpolated to $\lambda_{k}$ and $\lambda_{j}$. A linear interpolation is sufficient in this case because of the high sampling of $I_{\text {ref }}$. Various tests have shown that for the UVN instrument the HSM method results - at least in the UV-VIS - in the smallest errors when applying a retrieval to the reflectances.

If the impact of the inhomogeneous slit illumination on the spectral calibration is not taken into account, this results in a spectral mismatch between the radiance and the irradiance spectrum which adds "pseudo noise" into the reflectance spectrum $L$. The introduced pseudo noise is in the order of a few tenth of a percent in the UV-VIS, which needs to be compared to the SNR requirements of this sensor type which is typically several 100 in the short-wave UV and several 1000 in the UV and visible spectral range. Depending on the amplitude and the spectral correlation of the pseudonoise, trace gas retrieval of weak absorbers is degraded if this effect is not taken into account adequately. Therefore, the impact of inhomogeneous slit illumination on trace gas retrievals in the UV-VIS as well as options to minimise this will be assessed.

\subsection{Error mapping}

The information content and error analysis approach is based on the optimal estimation retrieval scheme and performance assessment (see e.g. Rodgers, 2000). Optimal estimation combines the information from the measurement with apriori information of the parameter to be retrieved.

Instead of a full retrieval, an error mapping is performed. We assume a moderately linear problem (i.e. neglecting nonlinearities) to determine the errors. The formulas for this approach are given in Appendix C. As a-priori state, the simulated state of the atmosphere is used. The linearisation of the forward model is performed around this a-priori state. The forward and instrument model is used to simulate the a-priori radiance. Here, the instrument model is assumed to be insensitive to the inhomogeneity of the scene, i.e. we simulate a homogenous illumination of the slit and use the homogeneous ISRF to calculate the spectra from the mean radiance of the scene. The inhomogeneous ISRFs are then used in the instrument model to determine how the measurement of the radiance is disturbed by the inhomogeneous illumination of the slit. The difference between the erroneous radiance and the true radiance is then mapped to a difference between the true state (which is also the a-priori state) and the state a retrieval would determine from the erroneous radiance. This difference estimates the size of the systematic error we get from the inhomogenous illumination of the slit and therefore from the inhomogeneity of the scene.

The systematic error would appear in a retrieval as a bias. The precision of an optimal estimation retrieval is determined by the covariance of the radiance measurement (i.e. the noise) and constrained by the a-priori covariance. In this manuscript emphasis is placed on the minimisation of the systematic errors.

In the error mapping model, four trace gases are considered: $\mathrm{O}_{3}$ (fitting window $\left.305-330 \mathrm{~nm}\right), \mathrm{NO}_{2}(405-500 \mathrm{~nm})$, $\mathrm{SO}_{2}(308-325 \mathrm{~nm})$, and $\mathrm{HCHO}(337-360 \mathrm{~nm})$. For all quantities, the profiles of the scenario as specified in Tables 1 and 2 are used as a-priori with an associated error of $50 \%$.

Note that the error mapping is always performed for only one of the trace gases. It is assumed in the analysis that the atmospheric state is perfectly known for all parameters except the retrieved one. Potential impacts of scene inhomogeneity on other retrieval parameters are not considered in the context of the present study. The only instrumental effect taken into account is the inhomogeneous illumination of the slit. A small error for a geophysical parameter resulting from a single instrumental error does not necessarily mean that this parameter can be retrieved with the estimated error. A full error budget needs to be built up for all instrumental limitations, including errors, introduced by the imperfect knowledge of cloudiness, surface albedo or aerosol loading. Since such an error budget would depend on the actual retrieval method, it is beyond the scope of the current study. 


\section{Results}

Figure 6 shows the correlation between the estimated systematic (relative) errors for $\mathrm{O}_{3}, \mathrm{NO}_{2}, \mathrm{SO}_{2}$ and $\mathrm{HCHO}$ tropospheric columns resulting from inhomogeneous illumination to the cloud fraction (left panels) and to the reflectance ratio (right panels) without a correction (red marks) and after a spectral calibration has been applied to each individual radiance (blue marks). Note that, as mentioned in Sect. 2.5, the estimated errors only consider the effect of the inhomogeneous illumination of the instrument slit on the measured spectrum.

As can be seen from this figure, large errors are generally observed at high cloud fractions. Without correction there is a strong correlation between the reflectance ratio and the trace gas errors, which shows that the reflectance ratio is suitable for a characterisation of the inhomogeneity. With spectral calibration (using the instrument spectral response function for homogeneous illumination), the errors and also the correlation between errors and reflectance ratio are significantly reduced.

The derived spectral shifts are different for each ground pixel and also depend slightly on wavelength. The mean spectral shift for all ground pixels is close to zero, i.e. positive and negative shifts cancel on average. The mean absolute shift is about $0.005 \mathrm{~nm}$; the maximum absolute shift is about $0.04 \mathrm{~nm}$.

To further quantify the results a statistical analysis of the errors has been performed. The following quantities are determined for each gas:

- mean absolute relative error

- maximum absolute relative error

- standard deviation of the relative error

- correlation coefficient between the relative error and the reflectance ratio

These quantities are determined for the full set of 400 spatial pixels and also for a reduced set containing only ground pixels with cloud fractions smaller than $20 \%$. The latter is more representative for a real UVN data set, because for ground pixels with too high cloud fraction no reliable tropospheric columns can be determined.

The results are shown in Fig. 7. Corresponding histograms of error distributions with and without wavelength calibration are shown in Fig. 8.

The distribution of errors is rather symmetric around zero before the correction and becomes much narrower (and sometimes slightly asymmetric) when the wavelength calibration is applied. After wavelength calibration there is usually a strong peak around zero error.

Without wavelength calibration mean systematic errors of up to about $6 \%$ are possible; maximum errors even exceed $50 \%$ (in the case of $\mathrm{NO}_{2}$ ). The errors for $\mathrm{O}_{3}$ are generally
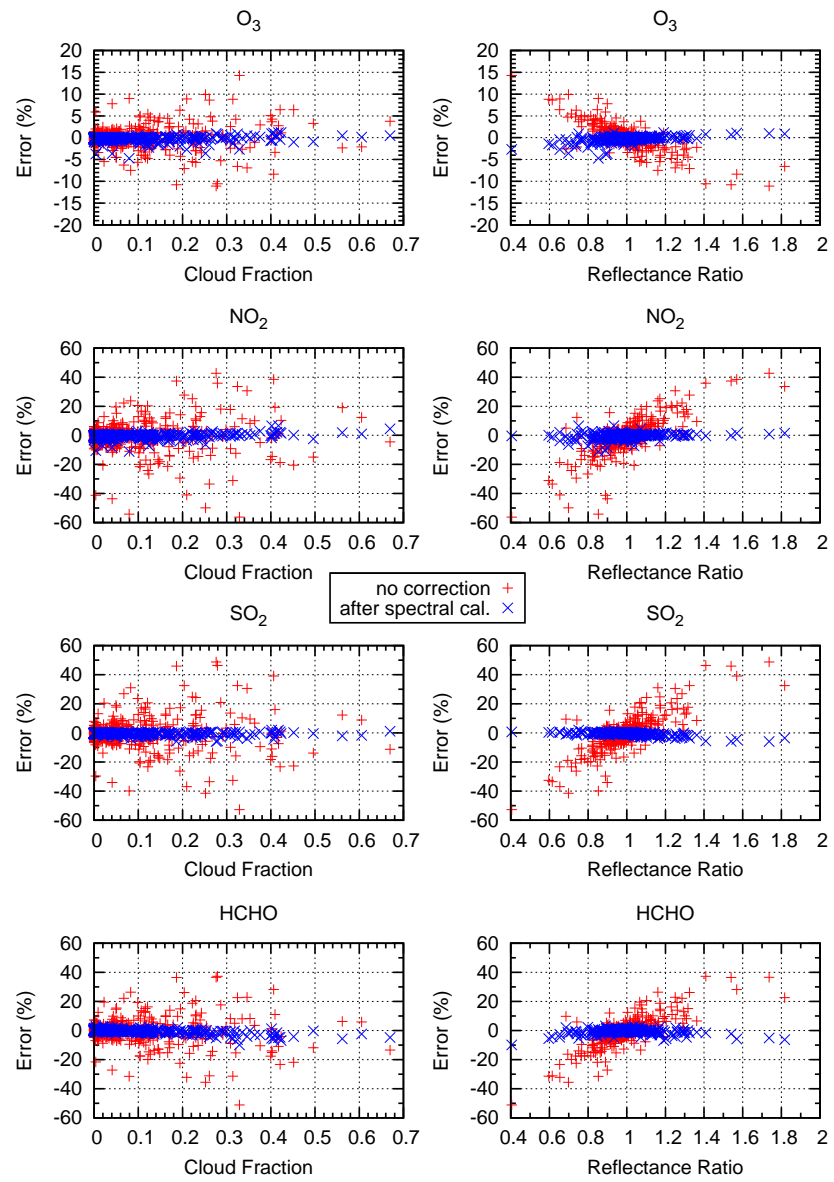

Fig. 6. Tropospheric column errors as function of cloud fraction (left panels) and reflectance ratio (right panels) without (red) and with (blue) spectral calibration.

smaller than for the other products. With wavelength calibration errors are largely reduced: Mean errors are $\sim 1 \%$ or smaller, maximum errors $\sim 10 \%$ or smaller. The standard deviation of the errors - which is a measure for the additional uncertainty of a derived product introduced by the inhomogeneous illumination conditions - is usually slightly larger than the mean absolute error and largely reduced when wavelength calibration is switched on. As already noticed before, the correlation of errors with the reflectance ratio is large without wavelength calibration and reduced afterwards. This is an indication that in the UV-VIS inhomogeneity is mostly compensated by the wavelength calibration. The main effect of the inhomogeneous illumination is the asymmetric ISRF (see Fig. 4), which results in an effective wavelength shift. This effect is corrected by the wavelength calibration. The second effect, the different shape of the ISRF, seems to play a minor role.

In Table 3, the main results after wavelength calibration and for cloud fractions up to $20 \%$ are summarised. In addition, an error reduction factor is given, which is defined as the ratio of the tropospheric column error (or standard deviation) 

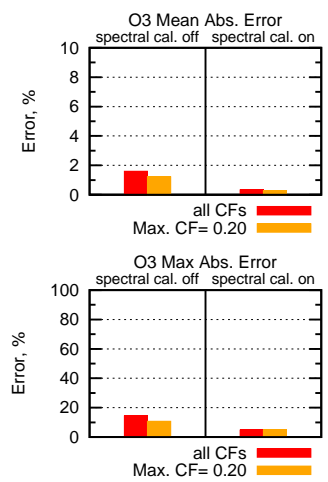

Max. $\mathrm{CF}=0.20$

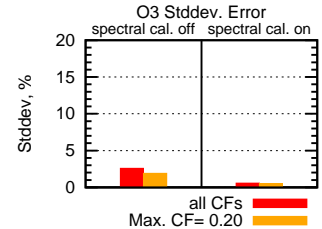

Max. $C F=0.20$

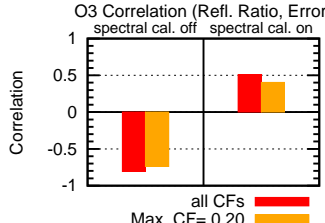

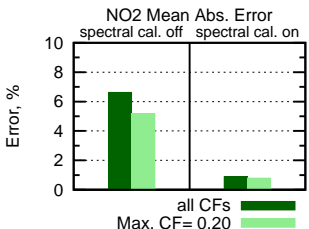

NO2 Max Abs. Erro

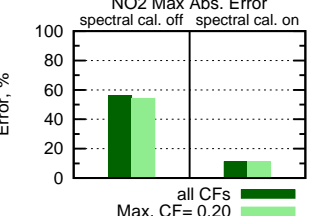

Max. $\mathrm{CF}=0.20$

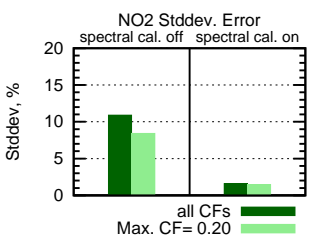

Max. $\mathrm{CF}=0.20$

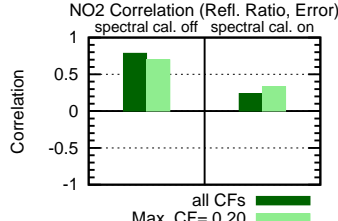

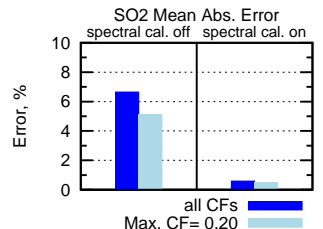

$\mathrm{SO} 2 \mathrm{Max}$ Abs. Error

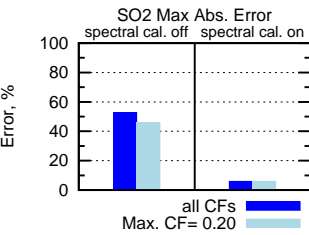

SO2 Stddev. Error

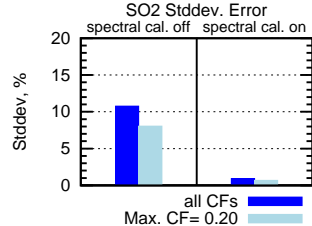

all $\mathrm{CFs}$
Max. $\mathrm{CF}=0.20$

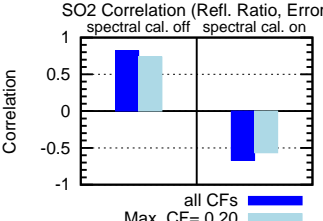

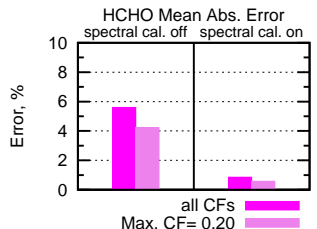

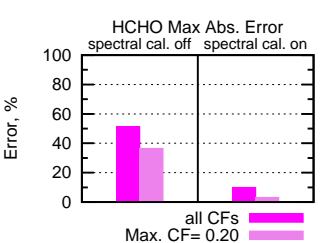

Max $C F=0.20$

$\mathrm{HCHO}$ Stddev. Error
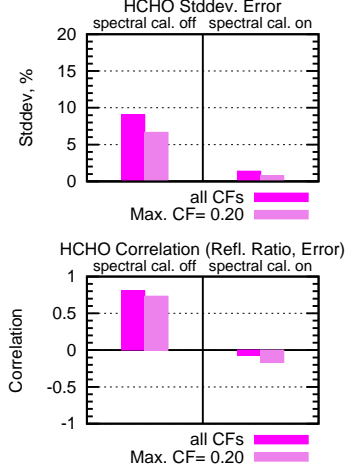

Fig. 7. Estimated errors of $\mathrm{O}_{3}$ (red/orange), $\mathrm{NO}_{2}$ (green), $\mathrm{SO}_{2}$ (blue) and $\mathrm{HCHO}$ (magenta). Top row: mean error; 2 nd row: maximum error; 3rd row: standard deviation of error; bottom row: correlation with the reflectance ratio. Values are given with and without spectral calibration, and for different maximum cloud fractions (CFs $100 \%$ and $20 \%$ ).

without corrections to the corresponding value after mitigation (i.e. wavelength calibration). The error reduction factor is especially high $(>10)$ for $\mathrm{SO}_{2}$ and $\mathrm{HCHO}$, where the retrievals are known to be very sensitive to spectral errors.

Although these results are quite promising, it should be kept in mind that they have been derived based on simulated data only. In the context of the present study, the inhomogeneous ISRFs are only used to estimate representative radiances from inhomogeneous scenes. In the spectral calibration and the error mapping, only the homogeneous ISRFs are used. For the simulations described here, this is sufficient to significantly reduce the errors of the tropospheric columns. However, the characteristics of the real instrument, which will be determined during on-ground calibration, will probably differ from the ones assumed in this study. Another aspect is that the actual retrieval method used to determine the tropospheric columns (in contrast to the simple error mapping approach used in the present study) might introduce additional uncertainties, e.g. due to limited knowledge of surface albedo or cloudiness and the inhomogeneity of these quantities over the observed scene. Therefore, it is recommended to repeat this analysis once the real instrument properties are known. If in this case the spectral calibration will turn out to be less efficient, additional mitigation strategies need to be considered. One of these strategies could be to estimate inhomogeneous ISRFs for a specific ground pixel based on sub-pixel information obtained in-flight during the scan. These ISRFs could then be used in the retrieval instead of the homogeneous ISRFs.

\section{Conclusions}

The impact of inhomogeneous illumination on Sentinel-4 UVN UV-VIS data products (tropospheric $\mathrm{O}_{3}, \mathrm{NO}_{2}, \mathrm{SO}_{2}$ and $\mathrm{HCHO}$ ) has been estimated based on simulated scenes. From the results presented above the following conclusions can be drawn:

- Inhomogeneity results in significant tropospheric column errors (up to about $5 \%$ mean error, $50 \%$ maximum error, $8 \%$ standard deviation of the errors) if no wavelength calibration is performed.

- With (good) wavelength calibration, the systematic error due to heterogeneous scenes is largely reduced (up to a factor of about 10 for the mean error, resulting in mean errors well below $1 \%$ and standard deviations of $1.5 \%$ or lower).

- The reflectance ratio is a good measure to characterise inhomogeneous illumination. The correlation coefficient between the reflectance ratio and the (uncorrected) errors of the retrieved products is \pm 0.7 . 

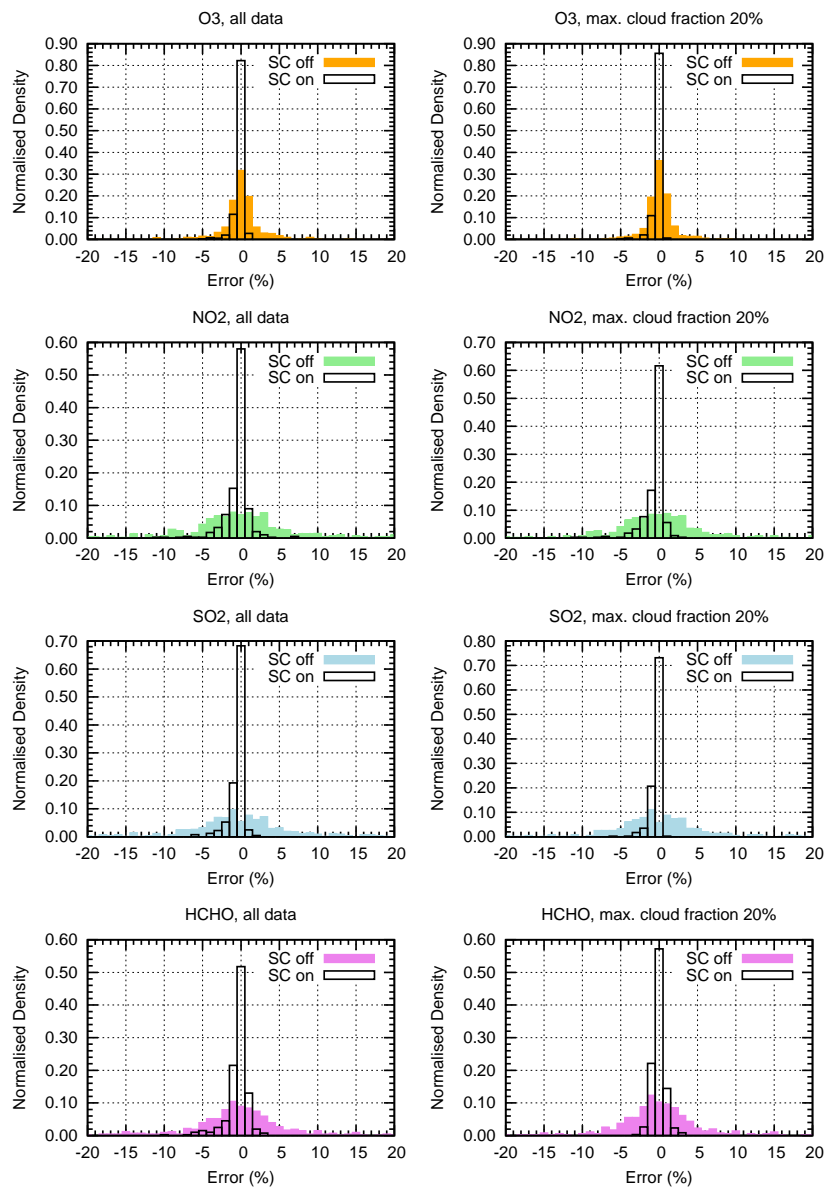

Fig. 8. Histograms of systematic errors due to scene inhomogeneity. The y-axis shows the number of sub-pixels with error in each bin (binsize 1\%), normalised to the total number of sub-pixels. Coloured boxes: without spectral calibration (SC); open boxes: with spectral calibration; left panels: based on all data; right panels: based on only data with maximum cloud fraction of $20 \%$.

The mean absolute errors and standard deviations of relative errors after spectral calibration are in the order of a few percent, which is about the estimated accuracy of the linear error mapping method. Thus, a wavelength calibration (performed for each radiance spectrum) seems to be sufficient to compensate the impact of inhomogeneous illumination.

However, all results presented here are based on simulated data for the UV-VIS band only. Therefore, it is recommended to determine representative ISRFs for inhomogeneous illumination during the on-ground calibration of UVN and to repeat the analysis described in this manuscript with these ISRFs and real measurement data and retrievals. In case larger errors are derived when using real measurement data, the retrievals could be further improved by taking into account inflight information on scene inhomogeneity and inhomogeneous ISRFs.

It should also be noted that we concentrate in this study on the impact and mitigation of inhomogeneous illumination
Table 3. Summary of results including wavelength calibration, taking into account only ground pixels with maximum cloud fraction of $20 \%$.

\begin{tabular}{lclr}
\hline & $\begin{array}{l}\text { No } \\
\text { correction }\end{array}$ & $\begin{array}{l}\text { After } \\
\text { wavelength } \\
\text { cal. }\end{array}$ & $\begin{array}{r}\text { Error } \\
\text { reduction }\end{array}$ \\
\hline & $\mathrm{O}_{2}$ & & \\
\hline Mean absolute rel. error & $1.2 \%$ & $0.3 \%$ & 4.4 \\
Maximum absolute rel. error & $10.8 \%$ & $4.7 \%$ & 2.3 \\
Standard deviation of rel. errors & $1.9 \%$ & $0.5 \%$ & 3.8 \\
Correlation coefficient & -0.7 & 0.4 & - \\
\hline & $\mathrm{NO}_{2}$ & & \\
\hline Mean absolute rel. error & $5.2 \%$ & $0.8 \%$ & 6.6 \\
Maximum absolute rel. error & $54.2 \%$ & $11.1 \%$ & 4.9 \\
Standard deviation of rel. errors & $8.4 \%$ & $1.5 \%$ & 5.8 \\
Correlation coefficient & 0.7 & 0.3 & - \\
\hline & $\mathrm{SO}_{2}$ & & 10.9 \\
\hline Mean absolute rel. error & $5.1 \%$ & $0.5 \%$ & 7.9 \\
Maximum absolute rel. error & $45.9 \%$ & $5.8 \%$ & - \\
Standard deviation of rel. errors & $8.1 \%$ & $0.7 \%$ & \\
Correlation coefficient & 0.7 & -0.6 & -4.4 \\
\hline & $\mathrm{HCHO}^{2}$ & & \\
\hline Mean absolute rel. error & $4.2 \%$ & $0.6 \%$ & \\
Maximum absolute rel. error & $36.5 \%$ & $3.2 \%$ & \\
Standard deviation of rel. errors & $6.7 \%$ & $0.8 \%$ & -0.2 \\
Correlation coefficient & 0.7 & & \\
\hline & & & \\
\hline
\end{tabular}

in across-slit (spectral) direction. For the UVN UV-VIS band this is sufficient, because there is from design almost no smile (i.e. no variation of spectral calibration in spatial direction). For other instruments or spectral bands, this might not be the case, such that also inhomogeneities in the along-slit (spatial) direction may have an impact. In the construction of new instruments, also hardware solutions to avoid inhomogeneous illumination of the slit using, e.g. spatial scrambler units as proposed by Gerilowski et al. (2011), should be considered.

\section{Appendix A}

\section{Derivation of inhomogeneous ISRFs}

The derivation of ISRFs is based on a general model of the spectral response function (SRF) for a dispersive spectrometer concept. The spectral dispersion is assumed to be perfectly aligned with the across-slit dimension of the spectrometer. Thus, the SRF model is reduced to this only dimension, and we understand the point spread function (PSF) hereafter as the along-slit integral of the two-dimensional spatial response. The across-slit dimension is labelled $x$ in slit coordinates and $X$ in object coordinates.

The following instrumental parameters are used in the calculations: 
- telescope PSF $\left(\mathrm{PSF}_{\mathrm{tel}}\right)$ in slit coordinates

- spectrometer PSF $\left(\mathrm{PSF}_{\mathrm{sp}}\right)$ in slit coordinates

- slit size $\Delta x_{\mathrm{S}}=45 \mu \mathrm{m}$

- slit size projection on Earth $\Delta X_{\mathrm{S}}=8 \mathrm{~km}$

- spectral oversampling factor $F_{\mathrm{OS}}(=3$ in the UV-VIS)

- spectral sampling step $(=1 / 6 \mathrm{~nm}$ in the UV-VIS $)$

Thus, the linear spectral dispersion factor with respect to the slit coordinates is approximated by $\Psi_{\lambda}=0.5 \mathrm{~nm} / 45 \mu \mathrm{m}$ in the UV-VIS. Currently, the PSFs are assumed to be wavelength independent. In the calculations the UVN PSF data sets for $500 \mathrm{~nm}$ (provided by ESA) are used. Note that the instrumental values assumed here correspond to the status during phase B1 of the UVN project in 2010.

\section{A1 Spectral response of a spatial subsample}

In order to account for the heterogeneous radiance field in the object space, it is necessary to consider the spectral response of discrete spatial subsamples $k$ in the across-slit dimension. In practice, the width of subsamples depends on the available information on illumination variation within a UVN spatial sample. In our case, the width $\Delta X_{k}$ in object space corresponds to the information derived from MODIS data at $500 \mathrm{~m}$ sampling. It is thus convenient to define the subsample width $\Delta x_{k}$ in slit coordinates by multiplication of $\Delta X_{k}$ with the ratio between slit size and spatial sampling distance $\Delta x_{\mathrm{s}} / \Delta X_{\mathrm{s}}$.

In a scheme from the entrance slit to the spectral detector, the spectral subsample response $\mathrm{SRF}_{k}$ is obtained as follows:

1. The subsample top-hat function (in the following represented by the symbol $\prod$ ) of width $\Delta x_{k}$ and centred on the subsample centre slit coordinate $x_{k}$ is convoluted (denoted by operator $\otimes$ ) with the telescope PSF $\left(\mathrm{PSF}_{\mathrm{tel}}\right)$. The result provides the normalised slit illumination in slit coordinates.

2. The normalised slit illumination of outer subsamples is partly out of the slit; this part is cut off by multiplication with a top-hat function of width $\Delta x_{\mathrm{s}}$, yielding the normalised slit illumination by sub-sample $k$ entering the spectrometer.

3. This illumination is convoluted with the spectrometer PSF $\left(\mathrm{PSF}_{\mathrm{sp}}\right)$, which results in the monochromatic detector illumination.

4. Convolution with the top-hat function of detector width (exit slit) size $\Delta x_{\mathrm{D}}=\Delta x_{\mathrm{S}} / F_{\mathrm{OS}}$ and subsequent conversion into spectral coordinates by application of the spectral dispersion factor $\Psi_{\lambda}$ yields the spectral response of subsample $k$ :

$$
\begin{gathered}
\operatorname{SRF}_{k}^{\prime}(\lambda+\delta \lambda)=\left(\left[\left(\prod\left(\frac{x-x_{k}}{\Delta x_{k}}\right) \otimes \operatorname{PSF}_{\text {tel }}(x)\right)\right.\right. \\
\left.\left.\prod\left(\frac{x}{\Delta x_{\mathrm{s}}}\right)\right] \otimes \operatorname{PSF}_{\mathrm{sp}}(x) \otimes \prod\left(\frac{x}{\Delta x_{\mathrm{D}}}\right)\right)\left(\frac{\delta \lambda}{\Psi_{\lambda}}\right) .
\end{gathered}
$$

Equation (A1) describes the spectral response of a slit subsample in case that the illumination of this subsample stays constant during the acquisition period. In a continuous scan mode, the object space is smeared by a scan motion distance $\Delta X_{\mathrm{s}}$. To compute subsample intensities (cf. Eq. A4 below), the scan motion has to be taken into account by smearing the MODIS radiance field at $500 \mathrm{~m}$ sampling with a onedimensional top-hat function of width $\Delta X_{\mathrm{s}}$. This solution is not optimum because the smearing process has to be applied to each geophysical scene the SRF model is applied to. Alternatively and equivalently, spatial subsamples can be defined in the object space, and represented over the dwell period in continuously progressing slit coordinates. The convenience is that input radiances do not have to be further processed. The scan motion is entirely (once and for all) taken into account by an additional convolution of sub-sample SRFs in slit coordinates with the motion smear function:

$$
\begin{array}{r}
\operatorname{SRF}_{k}(\lambda+\delta \lambda)=\left(\left[\left(\left\{\prod\left(\frac{x}{\Delta x_{\mathrm{s}}}\right) \otimes \prod\left(\frac{x-x_{k}}{\Delta x_{k}}\right)\right\} \otimes \operatorname{PSF}_{\text {tel }}(x)\right)\right.\right. \\
\left.\left.\prod\left(\frac{x}{\Delta x_{\mathrm{s}}}\right)\right] \otimes \operatorname{PSF}_{\mathrm{sp}}(x) \otimes \prod\left(\frac{x}{\Delta x_{\mathrm{D}}}\right)\right)\left(\frac{\delta \lambda}{\Psi_{\lambda}}\right) .
\end{array}
$$

We apply Eq. (A2) as SRF model for subsamples of width $500 \mathrm{~m}$ in object space.

\section{A2 Instrument spectral response function}

The sum of all $K$ non-zero subsamples $\mathrm{SRF}_{k}$ yields the total SRF in case of homogeneous slit illumination:

$\operatorname{ISRF}^{\text {hom }}(\lambda+\delta \lambda)=\sum_{k=1}^{K} \operatorname{SRF}_{k}(\lambda+\delta \lambda)$.

For perfect optics, $K$ would be 32 (slit size and smearing distance both correspond to 16 sub-samples or $8 \mathrm{~km}$ ). However, there is an additional broadening due to the PSF, which in the present case results in $K=36$.

This function is independent of geophysically driven illumination conditions and can be referred to as the instrument spectral response function (ISRF). Without any mitigation attempts, this function would be considered in the level 2 processing together with the measured spectra.

Heterogeneous illumination conditions within a spatial sample will modify the shape of the actual spectral response with respect to the ISRF. With $S_{k}$ being the intensity of subsample $k$ (in object space), the actual total spectral response is given by:

$\operatorname{ISRF}(\lambda+\delta \lambda)=\frac{\sum_{k=1}^{K} S_{k} \operatorname{SRF}_{k}(\lambda+\delta \lambda)}{\sum_{k=1}^{K} S_{k}}$. 
The homogeneous ISRF of Eq. (A3) is therefore a special case of Eq. (A4) with equal weights $S_{k}$.

In Eqs. (A1)-(A4), the sub-sample SRFs and the ISRF come out in arbitrary units. We define these arbitrary units such that the ISRF fulfils the normalisation condition:

$\int \operatorname{ISRF}(\lambda+\delta \lambda) \mathrm{d}(\delta \lambda)=1$.

\section{Appendix B}

\section{Spectral calibration algorithm}

The originally foreseen spectral calibration algorithm for UVN was based on a peak finding routine similar to the one used in the SCIAMACHY project, which uses the Falk (1984) algorithm. However, first tests with simulated UVN data showed that the accuracy of this algorithm is not sufficient to fulfil the UVN spectral stability requirements. Therefore, an alternative algorithm for spectral calibration has been developed.

The underlying assumptions for the algorithm are:

1. A first-guess wavelength calibration is available (e.g. from on-ground calibration) for the whole band, i.e. there should be an initial wavelength value associated to each spectral pixel.

2. The spectral variation of the wavelength calibration over the detector should be such that the "real" wavelength axis $\lambda_{\mathrm{R}}$ of $S$ can be described as a low-order polynomial function $P_{A}(\lambda)$.

The main idea of the algorithm is to determine the coefficients of $P_{A}$ by a non-linear least squares fit using the following equation:

$y(\lambda)=P_{B}(\lambda)+y_{\text {ref }}\left(P_{A}(\lambda)\right)$

where

$$
\begin{aligned}
y: & =\ln (s) \\
y_{\text {ref }}: & =\ln \left(S_{\text {ref }}\right) .
\end{aligned}
$$

Here, $S$ is a measured (irradiance or radiance) spectrum which is a function of wavelength $\lambda$, given of course at instrument spectral resolution and sampling. $S_{\text {ref }}$ denotes a spectrally well-calibrated (radiance or irradiance) reference spectrum that is a function of the "true" wavelength $\lambda_{R}$. A potential broadband radiometric offset between $S$ and $S_{\text {ref }}$ is taken into account by the polynomial $P_{B}$. Fit parameters are the coefficients of the polynomials $P_{A}$ and $P_{B}$. The resulting wavelength calibration is then given by:

$\lambda_{\mathrm{R}}=P_{A}(\lambda)$.

The fit is performed for the whole UV-VIS band in one go. This directly results in a wavelength calibration for the complete band.
The algorithm as described above is suitable for the wavelength calibration of irradiance spectra. However, a major challenge of the spectral calibration of radiances is the large dynamic range of possible radiances, which depend, e.g. on atmospheric absorption/scattering and surface albedo. As a consequence, there is a (wavelength dependent) intensity difference between the measured radiance $(y)$ and the reference spectrum $\left(y_{\text {ref }}\right)$, which usually can not be sufficiently compensated by the low order polynomial $P_{B}$.

The algorithm is able to handle these variabilities in the following ways, or by a combination of these, by:

1. Increasing the degree of the background polynomial $P_{B}$. Currently, a degree of 2 is used for irradiances and 12 for radiances.

2. An additional fit of spectral absorber features (i.e. of ozone in the UV-VIS), denoted with $\alpha$, which is defined as:

$$
\alpha(\lambda):=\frac{\partial y_{\mathrm{ref}}}{\partial c} c,
$$

where $c$ is the absorber amount (column) in absolute units and $\frac{\partial y_{\text {ref }}}{\partial c}$ is the absorber weighting function derived from radiative transfer calculations. Thus, $\alpha$ is essentially a normalised weighting function.

Considering $\alpha$ in the fit leads to the following slightly modified equation:

$y(\lambda)=P_{B}(\lambda)+y_{\mathrm{ref}}\left(P_{A}(\lambda)\right)+s \alpha\left(P_{A}(\lambda)\right)$,

where $s$ is a scaling factor which corresponds to a relative change of the absorber amount compared to the reference scenario. The usage of $\alpha$ in Eq. (B6) was inspired by the weighting function DOAS method (see, e.g. Coldewey-Egbers et al., 2005). As $y_{\text {ref }}, \alpha$ is determined with SCIATRAN.

Another problem for the spectral calibration of radiances is that the intensities of the radiances decrease rapidly towards the UV due to ozone absorption. If also the lower UV wavelengths are included in the fit, large uncertainties in the derived spectral calibration may occur. To reduce the impact of these wavelengths on the spectral calibration, the fit is performed using the error on the data as weights. Currently, this error is derived from signal-to-noise (see Fig. 5) only, but in-flight the end-to-end error could be used instead.

\section{Appendix C}

\section{Error mapping approach}

Here we describe in detail the analysis method for estimating the impact of systematic errors in the measured reflectances on the retrieved tropospheric trace gas columns. A quite general Bayesian/optimal estimation approach is used as described in more detail in (e.g. Rodgers, 2000). In the following we outline the error mapping approach in mathematical 
terms using a vector/matrix notation. The vector/matrix components correspond to:

- discrete wavelengths (mostly measurement detector pixel or channel centre wavelength)

- two altitude levels (troposphere, $0-10 \mathrm{~km}$ and stratosphere, $10-80 \mathrm{~km}$ ).

Let $\boldsymbol{x}$ be the vector of parameter of interest (e.g. the discretised $\mathrm{O}_{3}$ profile), $x_{\mathrm{a}}, \mathrm{S}_{\mathrm{a}}$ be the corresponding a-priori information (parameter vector and covariance matrix) and $c$ be the vector of other (assumed known) parameters (like atmospheric state/geolocation).

The high-resolution spectral reflectance $\boldsymbol{L}_{\text {high }}$ is computed via the radiative transfer model (RTM) $F$ (at sufficiently high spectral and vertical resolution):

$\boldsymbol{L}_{\mathrm{high}}=F(\boldsymbol{x}, \boldsymbol{c})$.

The (logarithm of the) simulated measured spectral reflectance $\boldsymbol{y}$ is then derived using the instrument model $M$ and a set of instrument parameters $i_{\mathrm{m}}$ :

$\boldsymbol{y}=M\left(\boldsymbol{L}_{\mathrm{high}}, i_{\mathrm{m}}\right)$.

The corresponding measurement error covariance matrix is denoted by $\mathbf{S}_{y}$. In the present case, the instrument model essentially contains the convolution of $\boldsymbol{L}_{\text {high }}$ with the ISRF and the application of the instrument sampling.

The weighting function matrix $\mathbf{K}$ is defined as

$\mathbf{K}:=\frac{\mathrm{d} \boldsymbol{y}}{\mathrm{d} \boldsymbol{x}}$.

Note that the forward model computes the weighting functions on a $1 \mathrm{~km}$ altitude grid. The weighting functions are then summed up over the relevant tropospheric and stratospheric sub-columns.

The measured (logarithm of the) reflectance for the a-priori scenario $\boldsymbol{x}_{\mathrm{a}}$ is given by:

$\boldsymbol{y}_{\mathrm{a}}=M\left(F\left(\boldsymbol{x}_{\mathrm{a}}, \boldsymbol{c}\right), i\right)$.

Here, $i$ denotes a set of instrument parameters assumed in the retrieval. This is not necessarily identical to the set $i_{\mathrm{m}}$ used in the calculation of the measured spectra. In the present case, different ISRFs are used in the calculation of $\boldsymbol{y}$ and $\boldsymbol{y}_{\mathrm{a}}$, namely inhomogeneous ISRFs for $\boldsymbol{y}$ and a homogeneous ISRF for $\boldsymbol{y}_{\mathrm{a}}$ (and also $\mathbf{K}$ ).

The measured (logarithm of the) reflectance for the actual scenario $\boldsymbol{x}$ is then approximated by:

$\boldsymbol{y} \approx \boldsymbol{y}_{\mathrm{a}}+\mathbf{K}\left(\boldsymbol{x}-\boldsymbol{x}_{\mathrm{a}}\right)$.

The solution vector (containing the retrieved parameters) is then given by
$\hat{\boldsymbol{x}}=\boldsymbol{x}_{\mathrm{a}}+\mathbf{G}\left(\boldsymbol{y}-\boldsymbol{y}_{\mathrm{a}}\right)$

with the retrieval (gain) matrix

$\mathbf{G}:=\frac{\mathrm{d} \hat{\boldsymbol{x}}}{\mathrm{d} \boldsymbol{y}}=\mathbf{S}_{\hat{\boldsymbol{x}}} \mathbf{K}^{T} \mathbf{S}_{\boldsymbol{y}}^{-1}$

and the solution error covariance matrix

$\mathbf{S}_{\hat{\boldsymbol{x}}}=\left(\mathbf{K}^{T} \mathbf{S}_{\boldsymbol{y}}^{-1} \mathbf{K}+\mathbf{S}_{\mathrm{a}}^{-1}\right)^{-1}$.

The systematic errors (in the present case those resulting from inhomogeneous illumination) are then given by the difference between the retrieved and true (a-priori) state vectors:

$\Delta \hat{\boldsymbol{x}}=\boldsymbol{x}-\boldsymbol{x}_{\mathrm{a}}=\mathbf{G}\left(\boldsymbol{y}-\boldsymbol{y}_{\mathrm{a}}\right)$.

The elements of $\Delta \hat{\boldsymbol{x}}$ are the errors of the tropospheric and stratospheric columns. The relative error is then defined as the ratio of the error derived this way to the true value.

Note that for some combinations of retrieved parameters and assumed errors, a subtraction of a polynomial in the retrieval improves the results. This is a standard technique for retrievals on real data. In this case, the state vector $\boldsymbol{x}$ will contain additional entries ( 3 in case of a second order polynomial) which are the coefficients of the polynomial as additional fit parameters.

Acknowledgements. MODIS data have been retrieved from the Level 1 and Atmosphere Archive and Distribution System (LAADS). This work has been performed in the context of the "In-flight Spectral Calibration and Requirements on Reflectance for Sentinel-4" study, ESA ESTEC Contract 4000101187/10/NL/MP/ef.

Edited by: F. Boersma

\section{References}

Ahlers, B., Bazalgette Courrèges-Lacoste, G., Guldimann, B., Veihelmann, B., Stark, H., and Dobber, M.: GMES Sentinel-4/UVN instrument concept and calibration approach, in: 20th CALCON Technical Conference proceeding, Logan, Utah, USA, 29 August-1 September 2011.

Bazalgette Courrèges-Lacoste, G., Ahlers, B., Guldimann, B., Short, A., Veihelmann, B., and Stark, H.: The Sentinel-4/UVN instrument on-board MTG-S, in: Proc. of 2011 EUMETSAT Meteorological Satellite Conference, 5-9 September 2011, EUMETSAT P. 59, Oslo, Norway, 2011.

Bovensmann, H., Burrows, J. P., Buchwitz, M., Frerick, J., Noël, S., Rozanov, V. V., Chance, K. V., and Goede, A. H. P.: SCIAMACHY - Mission Objectives and Measurement Modes, J. Atmos. Sci., 56, 127-150, 1999.

Bovensmann, H., Noël, S., Monks, P., Goede, A. H. P., and Burrows, J. P.: The Geostationary Scanning Imaging Absorption Spectrometer (GEOSCIA) Mission: Requirements and capabilities, Adv. Space Res., 29, 1849-1859, 2002. 
Bovensmann, H., Eichmann, K. U., Noël, S., Flaud, J. M., Orphal, J., Monks, P. S., Corlett, G. K., Goede, A. H. P., von Clarmann, T., Steck, T., Rozanov, V., and Burrows, J. P.: The geostationary scanning imaging absorption spectrometer (GeoSCIA) as part of the geostationary tropspheric pollution explorer (GeoTROPE): requirements, concepts and capabilities, Adv. Space Res., 34, 694-699, 2004.

Burrows, J. P., Weber, M., Buchwitz, M., Rozanov, V., LadstätterWeißenmayer, A., Richter, A., de Beek, R., Hoogen, R., Bramstedt, K., Eichmann, K.-U., Eisinger, M., and Perner, D.: The Global Ozone Monitoring Experiment (GOME): Mission Concept and First Scientific Results, J. Atmos. Sci., 56, 151-175, 1999.

Coldewey-Egbers, M., Weber, M., Lamsal, L. N., de Beek, R., Buchwitz, M., and Burrows, J. P.: Total ozone retrieval from GOME UV spectral data using the weighting function DOAS approach, Atmos. Chem. Phys., 5, 1015-1025, doi:10.5194/acp5-1015-2005, 2005.

Dobber, M., Voors, R., Dirksen, R., Kleipool, Q., and Levelt, P.: The High-Resolution Solar Reference Spectrum between 250 and $550 \mathrm{~nm}$ and its Application to Measurements with the Ozone Monitoring Instrument, Sol. Phys., 249, 281-291, doi:10.1007/s11207-008-9187-7, 2008.

Falk, W. R.: Data Reduction from Experimental Histograms, Nucl. Instrum. Meth. Phys. Res., 220, 473-478, 1984.

Gerilowski, K., Tretner, A., Krings, T., Buchwitz, M., Bertagnolio, P. P., Belemezov, F., Erzinger, J., Burrows, J. P., and Bovensmann, H.: MAMAP - a new spectrometer system for columnaveraged methane and carbon dioxide observations from aircraft: instrument description and performance analysis, Atmos. Meas. Tech., 4, 215-243, doi:10.5194/amt-4-215-2011, 2011.
Levelt, P., van den Oord, G., Dobber, M., Malkki, A., Visser, H., de Vries, J., Stammes, P., Lundell, J., and Saari, H.: The ozone monitoring instrument, Geoscience and Remote Sensing, IEEE T. Geosci. Remote, 44, 1093-1101, doi:10.1109/TGRS.2006.872333, 2006.

Perner, D. and Platt, U.: Detection of Nitrous Acid in the Atmosphere by Differential Optical Absorption, Geophys. Res. Lett., 6, 917-920, 1979.

Rodgers, C. D.: Inverse Methods for Atmospheric Sounding: Theory and Practice, Series on Atmospheric, Oceanic and Planetary Physics, vol. 2, World Scientific Publishing Co. Pte. Ltd., Singapore, 2000.

Rozanov, A., Rozanov, V., Buchwitz, M., Kokhanovsky, A., and Burrows, J. P.: SCIATRAN 2.0 - A new radiative transfer model for geophysical applications in the $175-2400 \mathrm{~nm}$ spectral region, Adv. Space Res., 36, 1015-1019, doi:10.1016/j.asr.2005.03.012, 2005.

Voors, R., Dobber, M., Dirksen, R., and Levelt, P.: Method of calibration to correct for cloud-induced wavelength shifts in the Aura satellite's Ozone Monitoring Instrument, Appl. Optics, 45, 36523658, 2006. 